\title{
Pulmonary mucormycosis in a patient with Crohn disease on immunosuppressive medications including infliximab
}

\author{
Alissa J Wright $M D^{1}$, Theodore Steiner $M D^{1}$, Ana Maria Bilawich $\mathrm{MD}^{2}$, John C English $\mathrm{MD}^{3}, \mathrm{C}$ Frank Ryan $\mathrm{MD}^{4}$
}

\section{CASE PRESENTATION}

A 68-year-old man presented to hospital with a two-day history of increasing shortness of breath, left-sided pleuritic chest pain and chills. His medical history was significant for longstanding Crohn disease ( $>55$ years) requiring multiple bowel resections in the 1970s in addition to high-dose corticosteroid therapy. For the past five years, he had been maintained on infliximab with only low-dose steroids for iatrogenic adrenal insufficiency. Additional medical problems included short gut syndrome, hypothyroidism and bronchiectasis; there was no history of diabetes. He experienced two hospital admissions in the preceding six months for pneumonia, one of which was due to Pneumocystis jirovecii confirmed by open lung biopsy. His medications on this admission included budesonide $6 \mathrm{mg}$ orally three times daily, azathioprine $100 \mathrm{mg}$ orally twice daily, infliximab $600 \mathrm{mg}$ intravenously every four weeks, levothyroxine $25 \mu \mathrm{g}$ orally daily, atovaquone $1500 \mathrm{mg}$ orally daily, and salbutamol and ipratropium inhalers as needed. His most recent dose of infliximab was one month before admission.

A physical examination revealed signs of consolidation in the left lung. His complete blood count included a white blood cell count of $15.2 \times 10^{9}$ cells/L, predominantly composed of neutrophils $\left(14.3 \times 10^{9}\right.$ cells $\left./ \mathrm{L}\right)$. Electrolytes were remarkable for a blood sugar level of $32.5 \mathrm{mmol} / \mathrm{L}$, with a negative screen for ketones. Arterial blood gas tests revealed a $\mathrm{pH}$ of 7.46 with a $\mathrm{CO}_{2}$ level of $26 \mathrm{mmol} / \mathrm{L}$. His albumin level was $18 \mathrm{~g} / \mathrm{L}$ (normal three months previously). A chest radiograph was suspicious for a cavity in the left upper lobe; a subsequent computed tomography scan of the chest confirmed a cavitary lesion measuring $10 \mathrm{~cm}$ in maximum diameter extending to the pleural surface, as well as extensive left upper and lower lobe consolidation (Figure 1A). He was diagnosed with recurrent pneumonia complicated by lung abscess formation and was started on piperacillin-tazobactam, azithromycin and stress-dose corticosteroids.

Sputum cultures on admission grew Klebsiella pneumoniae, while blood cultures were positive for Burkholderia cepacia. The patient was assessed by the infectious disease service, who recommended a change in the antibiotic regimen to meropenem as well as consideration of percutaneous drainage of the lung abscess. A serum hemoglobin $A_{1 c}$ level drawn three days after admission was $9.3 \%$ and the endocrinology service was consulted for management of new-onset diabetes mellitus.

Despite treatment, the patient continued to experience fluctuating shortness of breath and pleuritic chest pain. His peripheral white blood cell count remained elevated, and chest imaging showed no resolution of the lung abscess. A bronchoscopy was performed.

\section{DIAGNOSIS}

Bronchoscopy ruled out an endobronchial obstruction. Washings from the lingula were culture negative; however, cytology revealed the presence of fungal elements suspicious for Mucor/Rhizopus or Aspergillus species. Subsequent percutaneous drainage of the lung abscess was nondiagnostic. He underwent a left upper lobectomy that confirmed a lung abscess due to Mucor/Rhizopus species with pathological evidence of tissue invasion involving the chest wall and mediastinum (Figure 1B). Fungal cultures were ultimately negative.

Despite reduction in the patient's immunosuppression to maintenance corticosteroids and combination therapy with intravenous amphotericin and micafungin, the patient's condition deteriorated progressively and he died three months after the initial diagnosis of mucormycosis. Retrospectively, there was no exposure history, including exposure to hospital construction, which could account for his
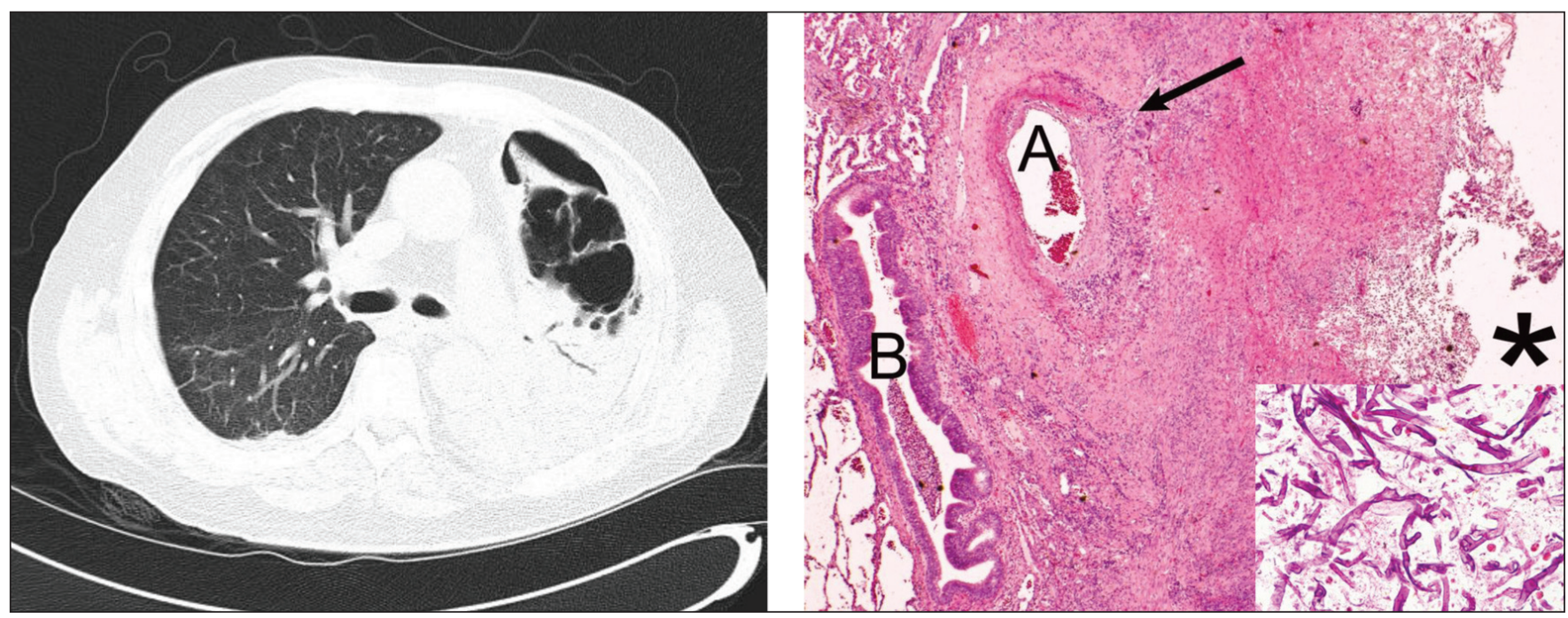

Figure 1) Left panel Computed tomography image of the chest (lung window) showing extensive consolidation of the left lung with cavitation extending to the pleural surface. Right panel Photomicrograph of one region of the cavity showing the surrounding rim of necrotic lung tissue. A prominent pulmonary artery (A) is partially necrotic and infiltrated by fungal hyphae (asterisk: cavity lumen; B: bronchiole). Inset: High-power photomicrograph of the hyphae of the Mucor/Rhizopus species. The hyphae are pauciseptate, of variable thickness and demonstrate a 'folded tissue' or 'ribbon-like' appearance. Some of the branches can be seen originating at right angles from the parent structure

${ }^{1}$ Department of Medicine, Division of Infectious Disease; ${ }^{2}$ Department of Radiology; ${ }^{3}$ Department of Pathology; ${ }^{4}$ Department of Medicine,

Respiratory Division, University of British Columbia, Vancouver, British Columbia

Correspondence: Dr Alissa J Wright, Massachusetts General Hospital, Infectious Disease Division, 55 Fruit Street, GRJ-504, Boston,

Massachusetts 02114-2696, USA. Telephone 617-726-3906, fax 617-726-7416, e-mail alissa.wright@ubc.ca 
infection. Although his ferritin level was $2238 \mathrm{pmol} / \mathrm{L}$ in the month before hospital admission, there was no evidence of iron overload, with a serum iron level of $12 \mu \mathrm{mol} / \mathrm{L}$ and iron saturation of $25 \%$.

\section{DISCUSSION}

Mucormycosis refers to a number of deep-seated invasive infections caused by fungi in the order Mucorales. These pauciseptate fungi are found ubiquitously throughout the environment but evoke a strong immune response in the normal healthy host, protecting the majority of exposed individuals from developing disease (1). It is only after the immune system has become compromised that invasion and disease can occur. Patients at highest risk are individuals with uncontrolled diabetes mellitus or prolonged neutropenia; those taking high-dose corticosteroid therapy; or those who are hemodialysis dependent and require iron chelation therapy with deferoxamine (2). In our patient, his newly detected diabetes would have contributed to his infection risk because phagocytes in a hyperglycemic environment are known to exhibit impaired chemotaxis and killing responses to Mucorales spores and hyphae (1).

Previously a rare condition, increases in the incidence of mucormycosis have been documented in both developed and developing countries (2). This change has been postulated to be due to a growing population of immunosuppressed patients, changes in medical treatment or prophylaxis for those at the highest risk for opportunistic infection, or improvement in diagnostic techniques for organisms that are traditionally difficult to culture (2). Mucorales are fragile and fragmentation of their large branching hyphae can render specimens nonviable. As a result, specimens that are not processed via grinding but instead either treated with a process known as 'stomaching' to homogenize the tissues or sliced yield better recovery (3).

The antitumour necrosis factor (TNF) agents, including infliximab, are potent immunosuppressive medications that are licensed for the treatment of a variety of autoimmune diseases including Crohn disease (4). Postmarketing surveillance has detected that patients treated with these drugs are at risk of a number of opportunistic infections. Reactivation tuberculosis was initially the primary opportunistic infection of concern because TNF is important in the formation and maintenance of granulomas $(5,6)$. However, TNF has other roles in the host defence system - including inducing phagosome activation and recruiting neutrophils and macrophages to the site of infection and, over time, there has been an expansion in the 'black box warning' for patients taking anti-TNF agents to include other opportunistic pathogens such as Listeria monocytogenes $(6,7)$. Although the increased risk is predominantly for well-known intracellular pathogens, it is not currently clear whether other pathogens, such as Mucorales, also pose a specific risk.

Even with the advent of newer treatment regimens for fungi in general, mucormycosis still portends a dismal prognosis (8). Effective therapy relies on a combination approach with reduction in immunosuppression and aggressive debridement at the site of infection paramount to cure. Medical treatment is more limited because Mucorales are intrinsically resistant to most of the available antifungals.

Amphotericin is considered to be the first-line agent for treatment, with multiple uncontrolled studies suggesting that liposomal preparations are superior to conventional deoxycholate (2). This may be because patients often require higher doses (ie, $5 \mathrm{mg} / \mathrm{kg} /$ day to $10 \mathrm{mg} / \mathrm{kg} /$ day) than needed for fungi such as Aspergillus species, and liposomal amphotericin is better tolerated. Posaconazole - the only triazole with activity against Mucorales - can be used as salvage therapy and has demonstrated benefit in small series (2). Because it is only available as an oral suspension, absorption can be highly variable among patients but improves with concomitant food intake (9). There is a theoretical risk of antagonism if these two agents are combined; however, combination therapy is sometimes used for exceedingly ill patients.
Echinocandins are not active against Mucorales. However, Rhizopus oryzae does possess the target enzyme for echinocandins and has been tried as a therapy in combination with amphotericin. A single retrospective study involving 41 patients with disseminated disease (10) suggested a survival benefit, although additional data are necessary to confirm these findings. In our patient, combination therapy with an echinocandin was chosen because the patient was already known to have a short gastrointestinal tract with potential absorption issues and, furthermore, was experiencing ongoing issues with gastrointestinal bleeding and diarrhea throughout his hospital stay.

We acknowledge that our patient had multiple risk factors that contributed to an overall high net state of immunosuppression. He was on high-dose azathioprine, had newly diagnosed diabetes mellitus and had been pulsed with steroids during his admission. However, we believe this case adds to the literature demonstrating the risk of opportunistic infections other than tuberculosis in patients undergoing antiTNF therapy. These infections can be remote from the site of the underlying disease process that necessitated anti-TNF therapy and can be exceedingly difficult to diagnose. In a patient receiving an antiTNF agent, such as infliximab or multiple immunosuppressants, failure to respond to conventional management for pneumonia or lung abscess should prompt careful reconsideration of all potential pathogens including angioinvasive fungi such as Aspergillus and the Mucorales. Recognizing the difficulty of culturing these organisms from conventional microbiological specimens, early consideration of invasive diagnostic procedures, such as percutaneous or open biopsy, is warranted.

\section{REFERENCES}

1. Kontoyiannis DP, Lewis RE. Agents of mucormycosis and entomophthoramycosis. In: Mandell GL, Bennett JE, Dolin R, eds. Mandell, Douglas, and Bennett's Principles and Practice of Infectious Diseases, 7th edn. Philadelphia: Churchill Livingstone Elsevier, 2010:4028, xcvii.

2. Sun HY, Singh N. Mucormycosis: Its contemporary face and management strategies. Lancet Infect Dis 2011;11:301-11.

3. Ribes JA, Vanover-Sams CL, Baker DJ. Zygomycetes in human disease. Clin Microbiol Rev 2000;13:236-301.

4. van Dullemen HM, van Deventer SJ, Hommes DW, et al. Treatment of Crohn's disease with anti-tumor necrosis factor chimeric monoclonal antibody (cA2). Gastroenterology 1995;109:129-35.

5. Gomez-Reino JJ, Carmona L, Valverde VR, Mola EM, Montero MD; BIOBADASER Group. Treatment of rheumatoid arthritis with tumor necrosis factor inhibitors may predispose to significant increase in tuberculosis risk: A multicenter active-surveillance report. Arthritis Rheum 2003;48:2122-7.

6. Koo S, Marty FM, Baden LR. Infectious complications associated with immunomodulating biologic agents. Infect Dis Clin North Am 2010;24:285-306.

7. U.S. Food and Drug Administration. FDA drug safety communication: Drug labels for the tumor necrosis factor-alpha (TNF $\alpha$ ) blockers now include warnings about infection with legionella and listeria bacteria. Online: U.S. Department of Health and Human Services; 2011. <www.fda.gov/Drugs/DrugSafety/ ucm270849.htm> (Accessed December 1, 2011).

8. Roden MM, Zaoutis TE, Buchanan WL, et al. Epidemiology and outcome of zygomycosis: A review of 929 reported cases. Clin Infect Dis 2005;41:634-53.

9. Li Y, Theuretzbacher U, Clancy CJ, Nguyen MH, Derendorf H. Pharmacokinetic/pharmacodynamic profile of posaconazole. Clin Pharmacokinet 2010;49:379-96.

10. Reed C, Bryant R, Ibrahim AS, et al. Combination polyenecaspofungin treatment of rhino-orbital-cerebral mucormycosis. Clin Infect Dis 2008;47:364-71. 


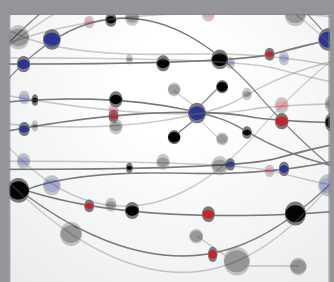

The Scientific World Journal
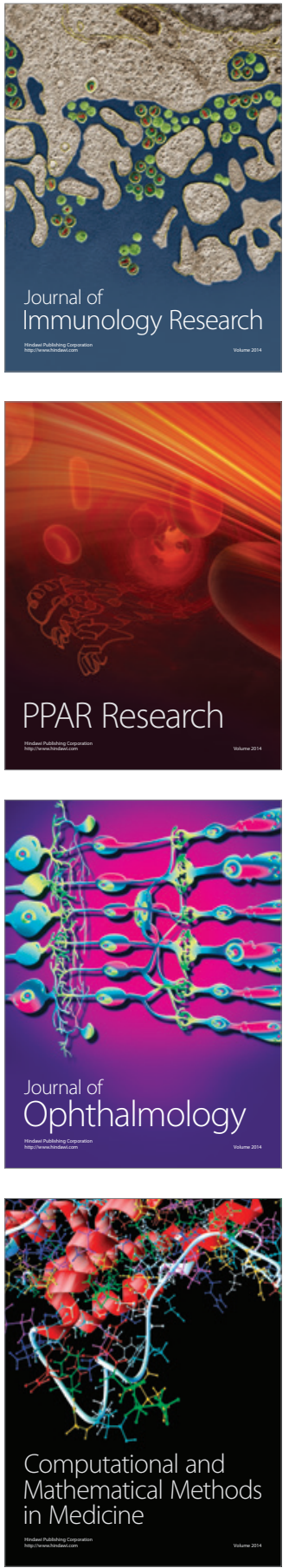

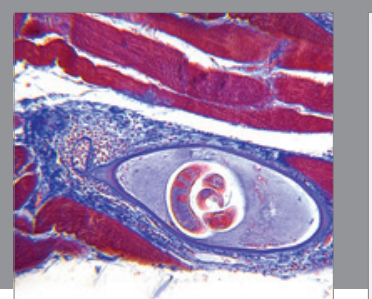

Gastroenterology Research and Practice

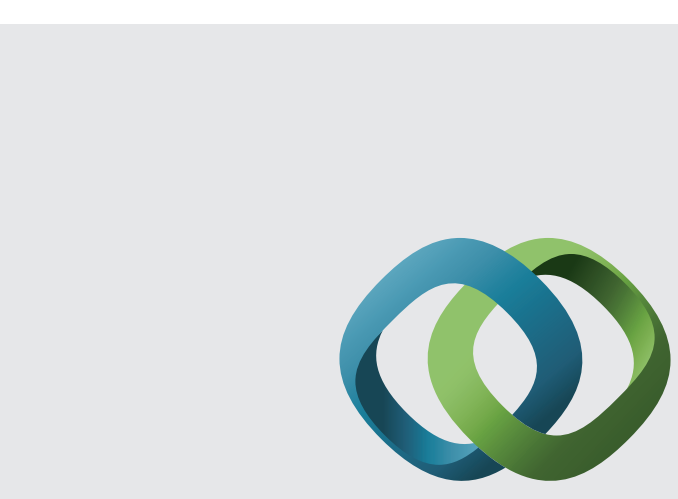

\section{Hindawi}

Submit your manuscripts at

http://www.hindawi.com
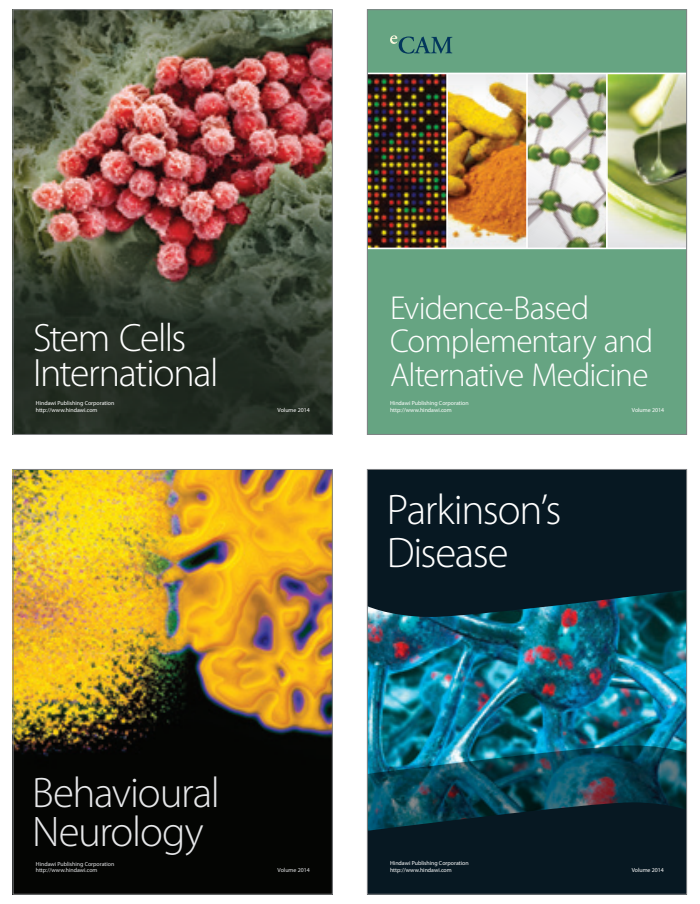
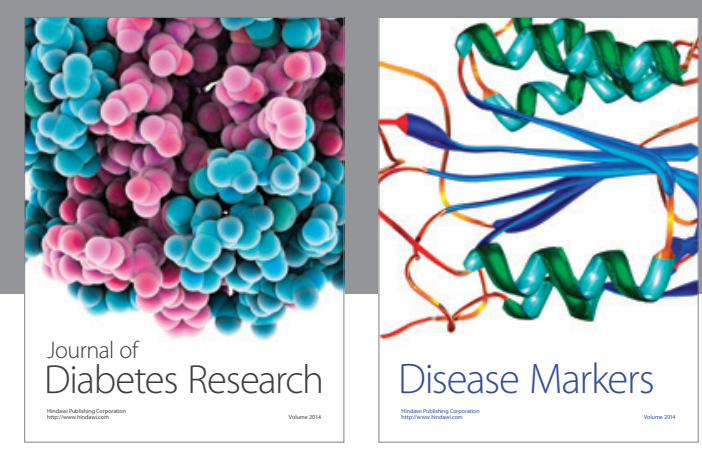

Disease Markers
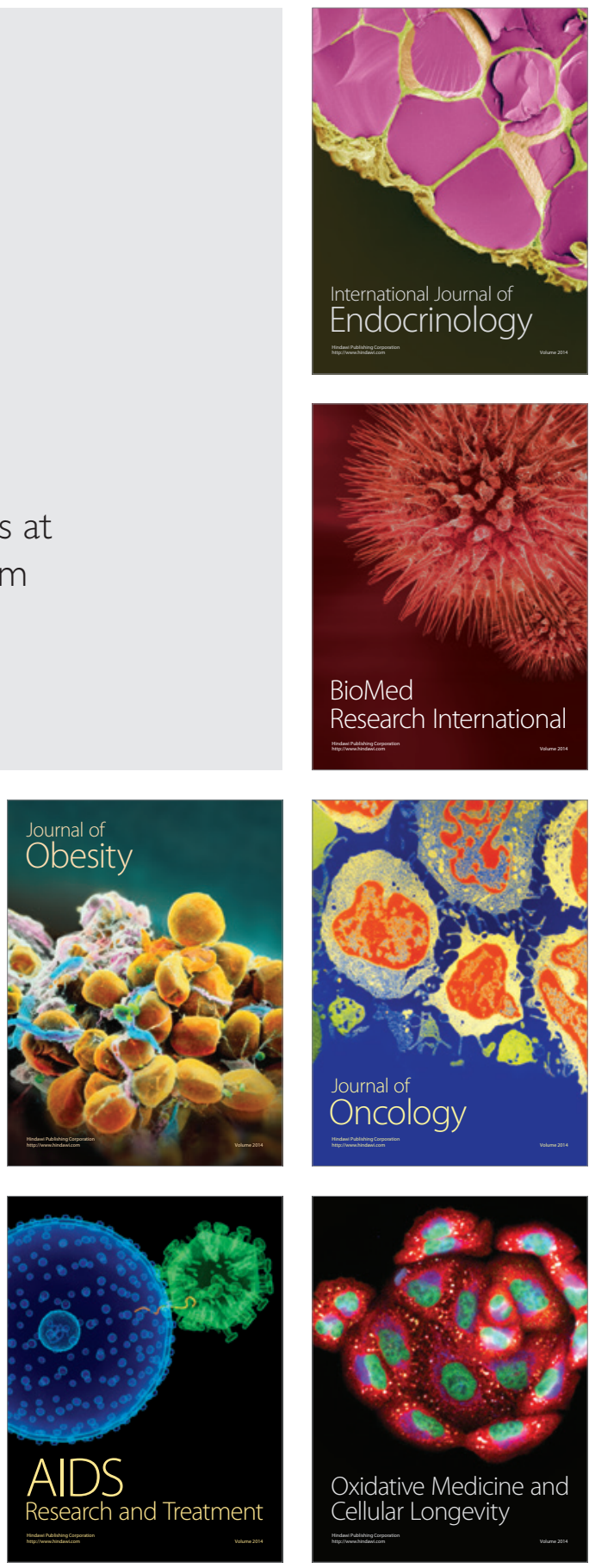